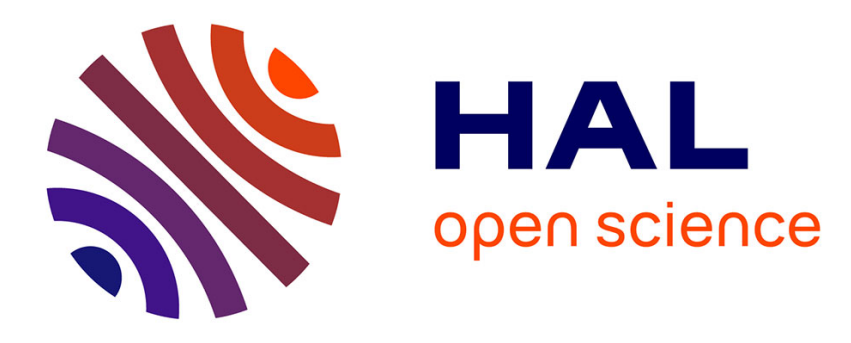

\title{
Sur le frottement intérieur observé a températures moyennes dans un aluminium de haute pureté
}

\author{
J. Friedel
}

\section{To cite this version:}

J. Friedel. Sur le frottement intérieur observé a températures moyennes dans un aluminium de haute pureté. Journal de Physique Lettres, 1978, 39 (5), pp.61-62. 10.1051/jphyslet:0197800390506100 . jpa-00231443

\section{HAL Id: jpa-00231443 \\ https://hal.science/jpa-00231443}

Submitted on 1 Jan 1978

HAL is a multi-disciplinary open access archive for the deposit and dissemination of scientific research documents, whether they are published or not. The documents may come from teaching and research institutions in France or abroad, or from public or private research centers.
L'archive ouverte pluridisciplinaire HAL, est destinée au dépôt et à la diffusion de documents scientifiques de niveau recherche, publiés ou non, émanant des établissements d'enseignement et de recherche français ou étrangers, des laboratoires publics ou privés. 


\title{
LE JOURNAL DE PHYSIQUE-LETTRES
}

\section{Classification}

Physics Abstracts

$61.70 \mathrm{~L}$

\section{SUR LE FROTTEMENT INTÉRIEUR \\ OBSERVÉ A TEMPÉRATURES MOYENNES \\ DANS UN ALUMINIUM DE haUTE PURETÉ}

\author{
J. FRIEDEL \\ Laboratoire de Physique des Solides (*), Université Paris-Sud, Centre d'Orsay, \\ 91405 Orsay, France
}

(Reçu le 21 novembre 1977, accepté le 11 janvier 1978)

\begin{abstract}
Résumé. - Le fort pic de frottement intérieur observé aux températures moyennes dans un aluminium de haute pureté est attribué au déplacement par déviation des nœuds des parois de polygonisation. Des expériences pourraient vérifier cette proposition.
\end{abstract}

\begin{abstract}
The strong peak of internal friction observed at medium temperatures in a high purity aluminium is attributed to the shift by cross-slip of the nodes of polygonized walls. Further experiments could check this proposal.
\end{abstract}

Un pic large mais fort de frottement intérieur a été récemment étudié dans l'aluminium à gros gains de haute pureté, après déformation de l'ordre de $1 \%$ et recuit au-dessus de $400 \mathrm{~K}$. Ce pic est centré aux environs de $400 \mathrm{~K}$ aux fréquences de l'ordre du hertz. L'ênergie d'activation décroît à amplitude croissante et peut se mettre sous la forme [1] :

$$
E=U-\sigma v
$$

où $\sigma$ est la contrainte appliquée et

$$
\begin{aligned}
U & =0,97 \pm 0,07 \mathrm{eV} \\
v & \simeq 750 b^{3} .
\end{aligned}
$$

Un pic analogue mais plus faible a été observé dans un aluminium moins pur, associé à des pics de plus forte énergie d'activation, et attribués au glissement le long des joints et à la montée de dislocations par diffusion de volume [2].

Les auteurs de l'observation sur l'aluminium pur remarquent que l'énergie $U$ est très voisine de celle mesurée par Murakani et Yoshida pour l'autodiffusion le long des lignes de dislocation [3]. Ils proposent que le fluage est dû à un glissement limité par la montée de crans, obtenue par diffusion de lacunes le long de la dislocation. Comme $2 v$ est le volume d'activation pour la contrainte moyenne résolue dans le plan de montée, $l=2 v / b^{2} \simeq 1500 b$ serait alors la distance moyenne entre crans le long des dislocations.
Ce processus n'est pas à rejeter a priori. Il semble cependant surprenant qu'il donne un pic aussi marqué ( $\delta$ supérieur à $10^{-1}$ sur plus de 100 degrés), qui correspond à une baisse de module très importante. Ce n'est en effet au mieux qu'une faible fraction des arcs de dislocation qui peuvent être gênés dans leur glissement par la présence de crans [4], comme le prouvent d'ailleurs les anomalies de module observées couramment dès de plus basses températures [5]. La distance de $1500 b$ semble d'autre part trop grande pour une distance entre crans à l'équilibre thermique à $400 \mathrm{~K}$ dans l'aluminium [6], et a fortiori après écrouissage et revenu. Finalement, aux fréquences du hertz, une lacune ne peut se déplacer le long d'une dislocation que sur une distance bien inférieure à $b v_{\mathrm{D}} \mathrm{e}^{-U / k_{\mathbf{B}} T}$ où $v_{\mathrm{D}} \simeq 10^{12} \mathrm{~s}^{-1}$. A $400 \mathrm{~K}$ avec $U \simeq 1 \mathrm{eV}$, cette distance limite est de $0,3 \mathrm{~b}$. Comme il y a nettement moins d'une lacune par distance interatomique à l'équilibre le long de la dislocation, la montée possible du cran est limitée à un ou deux $b$. Ceci est faible vis-à-vis des déplacements de glissement $\frac{l^{2}}{2 b} \varepsilon$ produits sur un arc de longueur $l$ par une déformation $\varepsilon$, aux déformations moyennes utilisées $\left(\varepsilon \simeq 10^{-5}\right)$, pour lesquelles ces déplacements sont de l'ordre de $10 b$; de plus l'effet de saturation à attendre à amplitude croissante ne semble pas observé.

On peut alors remarquer qu'un aluminium faiblement écroui et recuit a tendance à produire une polygonisation fine, et que le déplacement de ces parois de polygonisation est connu pour donner une forte baisse du module [7]. Il est donc tentant d'attribuer le pic de frottement intérieur observé vers $400 \mathrm{~K}$ à l'activation

(*) Laboratoire associé au C.N.R.S. 
thermique d'un déplacement supplémentaire des parois de polygonisation, par déplacement des noeuds de ces parois. Le facteur de concentration de contrainte $20 / b^{3} \simeq 1500$ serait alors le rapport au vecteur de Burgers $b$ de la taille des arcs de dislocation sur ces parois.

Deux processus de ce type peuvent $a$ priori être envisagés :

1) Montée des nœuds, par autodiffusion le long des dislocations. L'énergie $U$ mesurée semble en bon accord avec cette hypothèse. Elle se heurte à une même difficulté que celle de la montée des crans : aux fréquences du hertz, les nœuds ne pouvant au plus se déplacer que d'une distance interatomique, donnant un frottement intérieur faible et qui devrait se saturer à amplitude croissante.

2) Déplacement des nœuds par déviation thermiquement activée. Un processus de ce genre a été décrit par Washburn [8] et observé par Martin [9] en microscopie électronique à haute tension. Il doit nécessairement donner lieu à une énergie d'activation du type (1), avec une énergie $U$ de déviation sous contrainte nulle qui semble comparable aux estimations théoriques, certes grossières [10].

Plus exactement, du fait que les conditions de déviations ne sont pas nécessairement les mêmes que dans le fluage par déviation, les énergies $U$ mesurées dans les 2 cas doivent être voisines, mais pas nécessairement égales [11].

On peut en fait remarquer que l'analyse classique de l'énergie d'activation du fluage sous faibles contraintes faite par Dorn [12] fournit un palier intermédiaire très développé entre 400 et $600 \mathrm{~K}$, dont la valeur est comparable à $U_{1}$ quoiqu'un peu plus forte $(1,2 \mathrm{eV})$; elle est nettement plus faible que celle $(1,4 \mathrm{eV}) \mathrm{du}$ palier supérieur, attribué à la montée des dislocations par diffusion de volume. Les analyses plus récentes de Mythlyaev [13] ont montré que, dans ce domaine de température, l'énergie d'activation du fluage décroissait à contrainte croissante suivant une loi du type (1), avec pour l'aluminium une énergie $U$ comparable et une distance $2 v / b^{2}$ de l'ordre de la longueur des arcs de dislocation dans les parois de polygonisation formées lors du fluage.

Il est bon de rappeler que Dorn lui-même attribuait le stade de fluage considéré au processus de déviation. Cette interprétation, universellement acceptée à l'époque, a été quelque peu oubliée par la suite. Elle semble toujours la plus convaincante [11].

L'interprétation suggérée pour le pic de frottement intérieur suggère les vérifications expérimentales suivantes :

a) l'aluminium ultrapur de l'expérience décrite dans [1] est-il finement polygonisé ?

b) le volume d'activation $y$ est-il lié à la taille moyenne $l$ des axes de dislocation des parois de polygonisation par une loi de la forme

$$
2 v / b^{2} \simeq l
$$

comme dans les expériences de fluage décrites par Myshlyaev?

c) le simple décalage en température du pic, sans décroissance d'intensité, ou même une augmentation d'intensité sont-ils encore observés sur une plus grande gamme d'amplitude (ceci pour éliminer plus nettement la possibilité de friction par montée des nœuds de dislocation) ?

\section{Bibliographie}

[1] Esnouf, C., Gabbay, M. et Fantozzi, B., J. Physique Lett. 38 (1977) L 401.

[2] Woigard, J., Phil. Mag. 33 (1976) 623.

[3] Muraxami, H. et Yoshida, S., Cryst. Lattice Defects 6 (1975) 89.

[4] Friedel, J., Dislocations (Pergamon Press Londres) 1964, 199.

[5] Friedel, J., Phil. Mag. 44 (1953) 444.

[6] Friedel, J., Dislocations (Pergamon Press Londres) 1964, 309.
[7] Friedel, J., Boulanger, D. et Crussard, C., Acta Metall. 3 (1955) 380.

[8] Washburn, J., Appl. Phys. Lett. 7 (1965) 183.

[9] Martin, J. L., Colloque SFME Clermont-Ferrand (1976) sous presse.

[10] EsCaig, B., J. Physique 29 (1968) 225.

[11] FrIEDEL, J., Revue Phys. Appl. 12 (1977) 1649.

[12] DoRn, J. E. (1957), cf. [4], p. 305.

[13] Myshlyaev, M. M., cf. references [11]. 